\title{
Close approaches between Jupiter and quasars with possible application to the scheduled GAIA mission
}

\author{
J. Souchay ${ }^{1}$, C. Le Poncin-Lafitte ${ }^{2}$, and A. H. Andrei ${ }^{3}$ \\ 1 SYRTE, Observatoire de Paris, 61 Av. de l'observatoire, 75014 Paris, France \\ e-mail: Jean.Souchay@obspm.fr \\ 2 Lohrmann Observatory, Dresden Technical University, Mommsenstr. 13, 01062 Dresden, Germany \\ e-mail: christophe.le_poncin-lafitte@tu-dresden.de \\ 3 Observatorio Navional/MCT, Rio de Janeiro, Brasil Observatorio de Valongo/UFRJ, Rio de Janeiro, Brasil \\ e-mail: oat1@on.br
}

Received 29 September 2006 / Accepted 24 April 2007

\begin{abstract}
Aims. The recent improvements in ground and space observational techniques, as well as the launch of specific space missions such as Hipparcos, has considerably increased the precision of astrometric measurements. One of the most important goals still remaining is the link to the primary catalogue ICRF (International Celestial Reference Frame) and the dynamical reference determined through the time coordinates and the trajectories of moving celestial bodies, such as the Moon, the Sun, and the planets.

Methods. In this paper we investigate how this link can be made through the close encounters between Jupiter and quasars. We prefer to focus on the period involving the future space mission GAIA, and also to determine the corrections due to the relativistic deflection of the light of the quasars around Jupiter.

Results. After defining the $(\alpha, \delta)$ parallax effects between an Earth-based observational site and GAIA, we have obtained a large number of close encounters ( 232 between 2005 and 2015, with a threshold of 500"). Then in order to show typical configurations of close encounters, we selected several cases among the most interesting grazing ones, insisting on the relativistic deflection of light as seen from the two observational sites. We conclude that these events deserve a lot of attention in the future, in particular in the scope of a link between the dynamical reference system and the ICRF.
\end{abstract}

Key words. astrometry - reference systems - ephemerides

\section{Introduction}

Establishing an optimally accurate link between a quasi-inertial reference frame in space, such as the ICRF, and a frame to which the motion of celestial bodies is referred, such as the dynamical ecliptic of $\mathrm{J} 2000.0$, represents one of the most challenging aims of modern astrometry. While the ICRF (International Celestial Reference Frame), constructed from $S$ and $X$ bands VLBI observations, represents the best quasi-inertial reference frame, its counterpart at optical wavelengths is given by the positions and the proper motions at J2000.0 of the Hipparcos stars. Recently, the maintenance of the Hipparcos link has been continued through huge projects like the USNO CCD Astrograph Catalog (UCAC) whose the goal is to achieve a large completeness of the final improved catalogue for scheduled release in 2007. Moreover several investigations continued to use a substantial number of radio stars in order to link the optical frame to the ICRF (Boboltz et al. 2003; Fey et al. 2006). In this last paper, the positions of 46 radio stars were obtained in the ICRF directly through phase-referencing of the stars to nearby ICRF quasars. These positions were estimated to be accurate at the 10 mas level.

In a recent study Souchay et al. (2006) carried out the crossidentifications between the ICRF quasars of the most extended version of the ICRF, i.e. the ICRF-Ext.2 (Fey et al. 2004), and the 2003 version of the Véron-Cetty \& Véron optical catalogue (Véron-Cetty \& Véron 2003) of extragalactic objects, in order to evaluate the number of sources cross-identified at radio and optical wavelengths, and also to determine the optical photometric and redshifts characteristics of the ICRF.

In addition to these improvements, it is now well established that the quasars represent the most suitable objects for giving a quasi-inertial direction in space. This basic premise was at the origin of the ICRF (International Celestial reference Frame) consisting in a set of 212 radio sources whose equatorial coordinates with respect to the mean equator of J2000.0 are considered as fixed. VLBI observations have shown that, generally speaking, the quasars are such remote objects that despite their very high recession speed, their apparent proper motion is generally negligible. Only a rather small amount of sources show significant displacement of their photocenter at radio wavelengths (Fey et al. 2004).

Obviously one of the most valuable methods linking the dynamical system to the ICRF consists in directly observing the close encounters between the planets and a given quasar, supposed to be fixed with respect to the ICRF. Thanks to the extreme sensitivity of present CCD detectors and recent surveys like the SDSS (Sloan Digital Sky Survey, Schneider et al. 2002), the number of recorded quasars has considerably increased, and the compiled catalogue built by Véron-Cetty \& Véron (2003) contains 64866 extragalactic objects, among which 48921 are quasars. Consequently the chance of observing a close encounter between a quasar and a planet has considerably increased. In the following we will focus on this kind of event for the planet Jupiter. Notice that the 2003 version of the 
Véron-Cetty \& Véron (2003) catalogue is used instead of the updated 2006 version since the full release of the SDSS last phase (DR5), has not yet been fully processed at the time of writing this paper (Adelman-McCarthy et al. 2006). Under this provision it is not yet possible to derive the equivalent GAIA magnitudes in order to realistically filter out useless close encounters.

Notice that we think that our study is particularly well suited in the scope of the GAIA space astrometric mission. GAIA is planned as a survey mission and will make observations of roughly 1 billion objects. Among these objects we find that a large proportion of all known extragalactic reference sources are quasars. As already noticed by several authors (see for instance Fey et al. 2004), if the projected astrometric accuracies for GAIA are realized, the GAIA astrometric grid will be serious competition for the radio realization of the ICRF. Therefore any close encounter phenomena between a planet and known quasars is worthy of interest, not only during, but also before and after, the GAIA mission.

Our choice for the planet Jupiter is motivated by several reasons that we enumerate below:

- Jupiter is by far the most massive of the nine planets of the solar system, so that grazing close encounters between this planet and quasars, in addition to their fundamental astrometric interest, might lead to a significant and detectable relativistic effect, i.e. the deflection of the beam coming from the remote extragalactic object when its line of sight remains close to the limb of the planet. As detecting this effect should be a challenge for modern astrometry, we will illustrate in this paper how large this effect can be on several occasions and how it can significantly affect astrometric reductions at the level of several milliarcseconds. Moreover, second-order effects such as the influence of Jupiter's mass-quadrupole moment $J_{2}$ and also $J_{4}$, might be detectable from the GAIA upcoming space mission.

- The close approach between a quasar and Jupiter is automatically accompanied by a close approach with most of its satellites, since most of them lie in the equatorial plane of the planet. As more than 40 satellites have been detected in total, this obviously increases the interest of the study, because differential astrometry between the large Galilean satellites, as well as between the much smaller point-like ones, and quasiinertial objects such as quasars is a very efficient way to fit ephemerides and to contribute to the link between the dynamical system and the ICRF. The fact that the planet Jupiter, together with Saturn, owns a large number of satellites also multiplies the chances of very close approaches between these satellites themselves and a drastically increasing number of recorded quasars as astrometric reference objects for a quasiinertial system.

- An important study concerns the parallax effects one : Jupiter will be a priority target for the GAIA space mission, in particular for the two above reasons, then it is interesting to calculate the parallax angle precisely between the astrometric satellite and an Earth-based observer as seen from Jupiter (and reciprocally) both in a general situation and during close encounters between Jupiter and quasars. Note that simultaneous observations or observations with very small observational time intervals between the satellite and ground-based telescopes might bring interesting comparisons from an astrometric point of view.

We have no doubt that, in the near future, the number of recorded quasars per field of view with very precise equatorial coordinates with respect to the ICRF will allow quasars to play exactly the same role as the stars that now play the role of astrometric standards, but with the big adavantage that they do not present significant proper motions on long time scales (one century).

As mentioned above, the relative proximity between quasars and Jupiter's satellites during close approaches looks like a very efficient way to establish the link between the ICRF and the dynamical frame. The method should be the following one. On one hand precise ephemerides of the satellites enable one to theoretically give the exact location of the planet's center of mass with respect to the satellites. On the other hand differential astrometry between one or several satellites and the quasar during the close approach through large telescopes or GAIA should bring an optimal determination of the differential position between these moving objects and the quasar. The measurements should be all the more efficient because the very small angular size of Jupiter's satellites (especially the small ones) leads to a very accurate determination of their photocenter. Combining these two determinations on a theoretical and observational basis should lead to very precise determination of the position of Jupiter center of mass with respect to the inertial direction represented by the quasar. A repetition of these observations with a large number of quasars should finally lead to very accurate ephemerides of Jupiter with respect to a quasi inertial reference frame.

To achieve these various goals, we determine the close encounters occurring between Jupiter and recorded quasars in the first part of this paper, insisting on the parallax effect between the Earth and the astrometric satellite GAIA from which such events should be detected, and giving details on the orientation of the polar axis of the planet (perpendicular to its equator) with respect to the line of sight. Then we take into account the relativistic light deflection due to the mass-monople and the massquadrupole moment of the Jovian gravitational field by using some approximations of a general formalism recently proposed by Le Poncin-Lafitte \& Teyssandier (2005).

\section{Statistics about close encounters between Jupiter and quasars}

Using the B1.0 catalogue constructed by the USNO (US Naval Observatory), we identified a large number of close encounters between Jupiter and quasars with good astrometric precision, for a period of time ranging between 2005.0 and 2015.0, which corresponds to a little less than a revolution of Jupiter around the Sun (11.6 years) and which includes the period of time related to the GAIA astrometric space mission, scheduled around 2010. The input list used for the selection of of quasars (Véron-Cetty \& Véron 2003) derives from different surveys that have been undertaken in only a few specific zones of the sky, such as the SDSS (Sloan Digita Sky Survey, Schneider et al. 2002) or the 2dF survey (Croom et al. 2001).

Figures 1 and 2 represent the variations in Jupiter's right ascension and declination respectively as seen from the Earth during the period involved, that is to say, between 2005.0 and 2015.0, together with the positions of the quasars that have been selected for the close encounters. Their number amounts to 232 candidates. These objects are far from being homogeneously distributed along Jupiter's trajectory for two reasons. At first the catalogue used for the selection of quasars comes from surveys that have been undertaken in only a few specific zones in the sky. At second a large depletion zone corresponds to the galactic plane, whose the thickness does not allow to extragalactic objects to be detected. Notice that the curve showing 


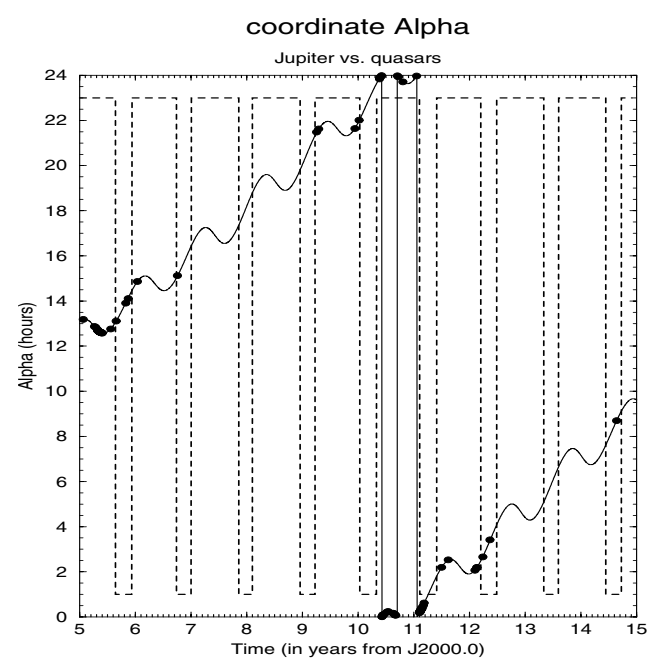

Fig. 1. Curve showing the evolution of Jupiter's right ascension $\alpha_{\mathrm{J}}$ (continuous curve), together with the right ascension of the quasars selected as candidates for a close encounter (bold circles). The dashed lines indicate the periods for which the observation is possible from GAIA (large horizontal segments).

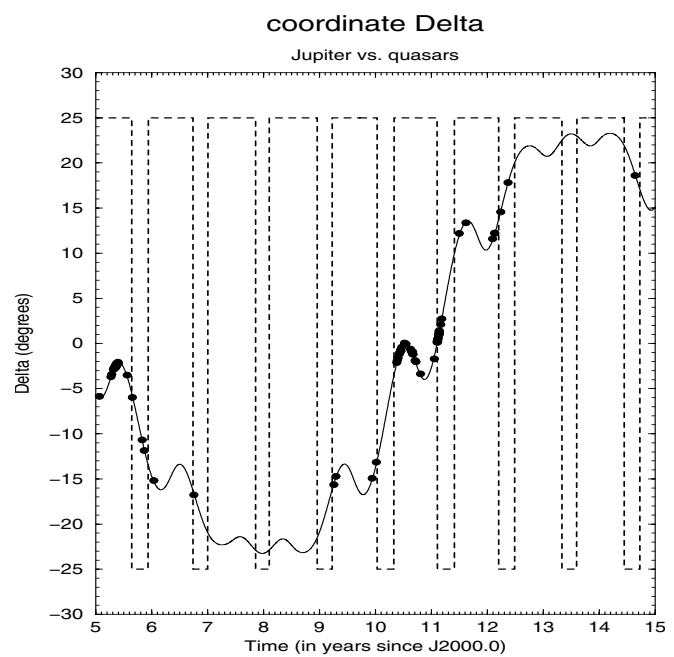

Fig. 2. Curve showing the evolution of Jupiter's declination $\delta_{\mathrm{J}}$ (continuous curve), together with the declination of the quasars selected as candidates for a close encounter (bold circles). The dashed lines indicate the periods for which the observation is possible from GAIA (large horizontal segments).

the evolution of the right ascension is characterized by a regular oscillation due to Jupiter's retrogradation at each synodic period of revolution (roughly 13 months). Notice also that only the zone between roughly $\alpha_{\mathrm{J}}=10 h$ and $\alpha_{\mathrm{J}}=12 \mathrm{~h}$ is not represented because Jupiter does not accomplish a whole revolution around the Sun during the 10-year time span considered. Jupiter's retrogradations also leads to oscillations of the declination $\delta_{\mathrm{J}}$ (Fig. 2), which ranges peak to peak to $\pm 25^{\circ}$ (Earth's obliquity is $23^{\circ} 26^{\prime}$ and Jupiter's inclination with respect to the ecliptic is close to $2^{\circ}$ ).

Although the GAIA mission will not occur before 2010, we carried out our study for the satellite starting from 2005, by extrapolating its orbit for the period 2005-2010. Notice that, for technical reasons, observations of Jupiter from GAIA will be possible only on condition that the lines joining the satellite to Jupiter's barycenter and to the Sun's barycenter will make an angle of at least $45^{\circ}$. This condition is shown in Figs. 1 and 2

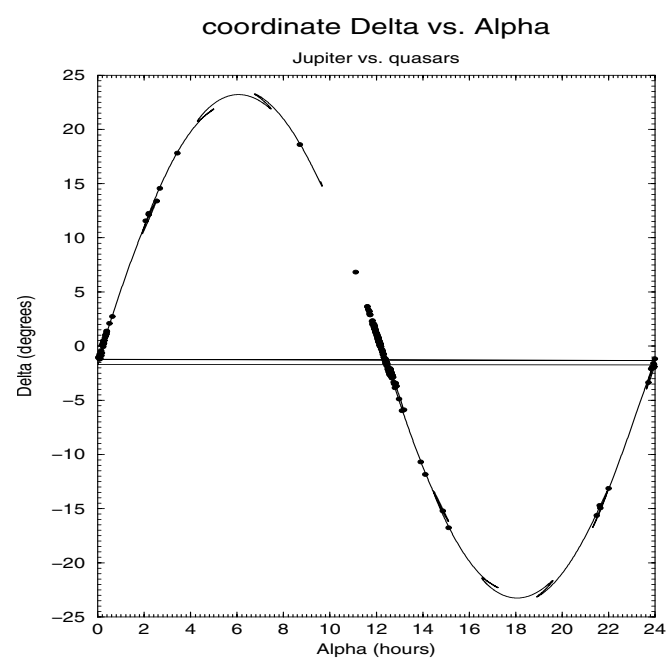

Fig. 3. Two-dimensional curve $\left(\alpha_{\mathrm{J}}, \delta_{\mathrm{J}}\right)$ representing the motion of Jupiter during the period 2005.0-2015.0) together with the location of the quasars chosen as candidate for a close encounter (black points).

whereas the small complementary segments correspond to conditions for which the observation is not possible. We notice that the majority of the observations correspond to the favorable case.

In Fig. 3 we show the two-dimensional $\left(\alpha_{\mathrm{J}}, \delta_{\mathrm{J}}\right)$ spatial curve described by Jupiter, together with the corresponding location of the quasars selected for close encounters. Notice that a majority of the quasars can be found in the right ascension interval $11 h<$ $\alpha<13 h$, which corresponds to the period 2010-2012, that is to say, roughly the beginning of the scheduled GAIA mission.

\section{Close encounters as seen from the Earth and from the GAIA satellite}

The improvement in ground-based techniques such as adaptative optics at the focus of large telescopes (Diolaiti et al. 2000) and very sensitive CCD detectors, might enable one to determine very accurately the angular distance between the photocenter of a planet like Jupiter and a given quasar, even very faint one (with apparent magnitudes greater than $V=18$ ), as is the case for a large majority of recorded quasars. In the continuity of recent very promising astrometric determinations at the level of 0.001" (Diolaiti et al. 2000), the performance of the observations of close encounters as measured from the Earth might be very valuable for linking the dynamical reference frame with respect to the ICRF implicitely represented by quasars even when they do not themselves belong to the ICRF catalogue.

This leads us to consider here close encounter events both from the Earth and from GAIA. In Fig. 4 we represent the curves of the differences in arcseconds between the equatorial coordinates of Jupiter as seen from the two observational sites (GAIA and the Earth) still for the time interval [2005-2015]. Although GAIA is scheduled to be launched after 2010, we extrapolated its orbital motion to use its fictitious position between 2005 and 2010.

As GAIA is moving along a Lissajous orbit with an osculating plane very close to the ecliptic, the differences $\Delta \delta$ are significantly smaller than the corresponding differences $\Delta \alpha$ in right ascension. As GAIA will be very closed to a Lagrange point, the two signals are characterized by a sinusoid with period of 1 year. The amplitudes reach $\pm 900^{\prime \prime}$ and $\pm 350^{\prime \prime}$. These values are considerably higher than Jupiter's apparent diameter, which does not exceed roughly $50^{\prime \prime}$. This means that the cases for which 


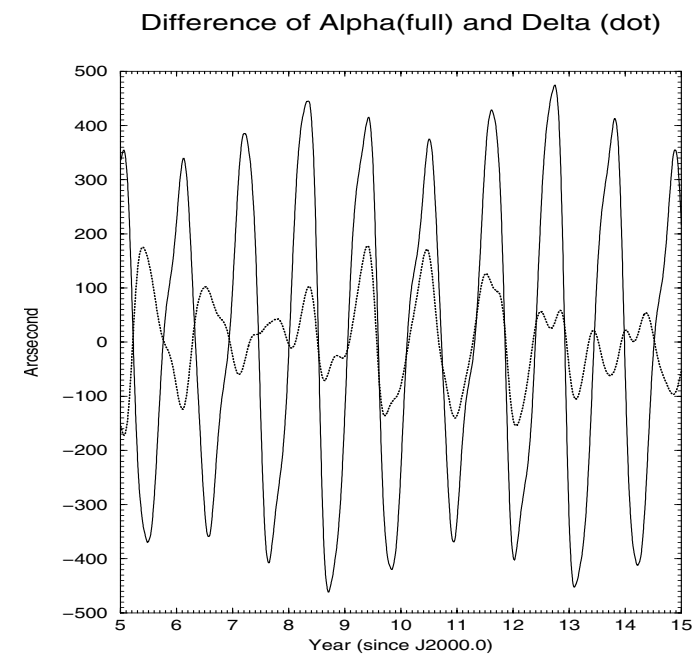

Fig. 4. Difference $\Delta \delta$ vs. $\Delta \alpha$ of Jupiter declination and right ascension as seen from the Earth and from GAIA.

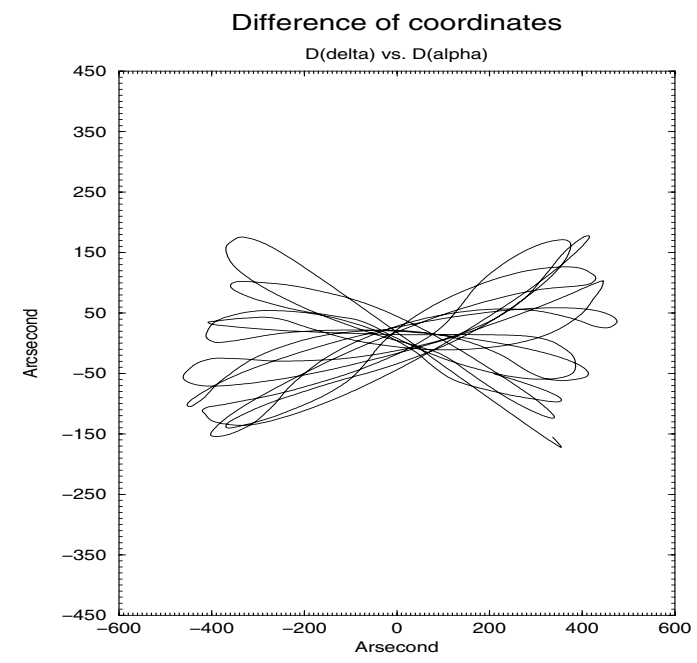

Fig. 5. Curve showing the difference in Jupiter's declination $\Delta \delta$ with respect to its corresponding difference in right ascension $\Delta \alpha$, when seen from the Earth and from the satellite GAIA.

the quasar that is subject to a close encounter is approaching the planet's limb above and beneath the planet according to the observer (on the Earth or from GAIA) might occur frequently, as well as the case for which a close encounter as viewed from the Earth corresponds to an occultation from GAIA or reciprocally. In Fig. 5 we represent the bi-dimensional parallax effect $\left.\left(\Delta \alpha_{\mathrm{J}}, \Delta \delta_{\mathrm{J}}\right)\right)$ during the same time interval as in Fig. 4.

In Figs. 6 and 7 we show the histograms giving the number of close encounters as seen from the Earth with respect to a given interval of $d_{\min }$, where $d_{\min }$ is the smallest angular distance during the close approach. The constant bins are 50 arcsec (Fig. 6) and 10 arcsec (Fig. 7), respectively. The number of quasars in Fig. 6 is quite stable, oscillating between 13 events (between $0^{\prime \prime}$ and $50^{\prime \prime}$ ) and 25 events (between $300^{\prime \prime}$ and 350"). In contrast, the feature of the histogram is much more chaotic when choosing the $10^{\prime \prime}$ interval (Fig. 7) with a number of events varying between 1 and 7 events.

In Tables 1 and 2 we show the characteristics of 24 of the most interesting close-encounter events occurring during the description interval of time between 2010.0 and 2012.5. Together with the equatorial coordinates of the quasars with respect to the ICRF (equator of J2000.0), we indicate the exact date of the

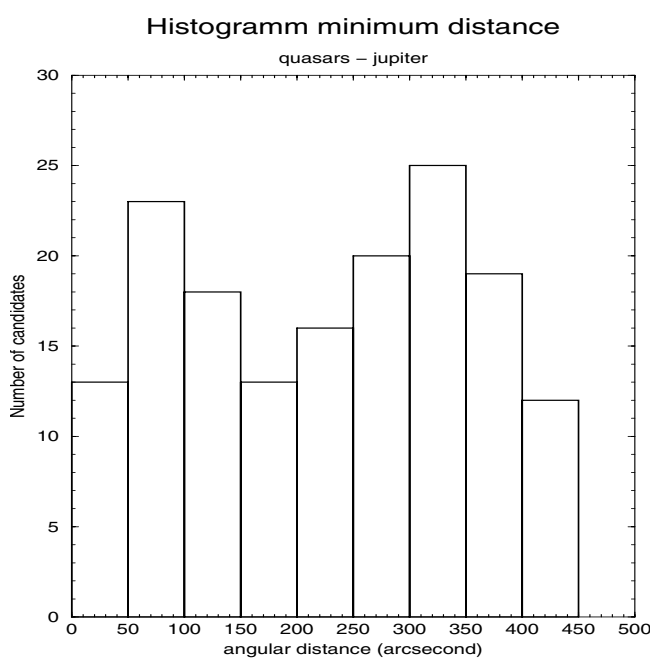

Fig. 6. Histogram showing the number of close approaches between Jupiter and quasars with respect to the minimum angular distance between Jupiter's center and the quasar.

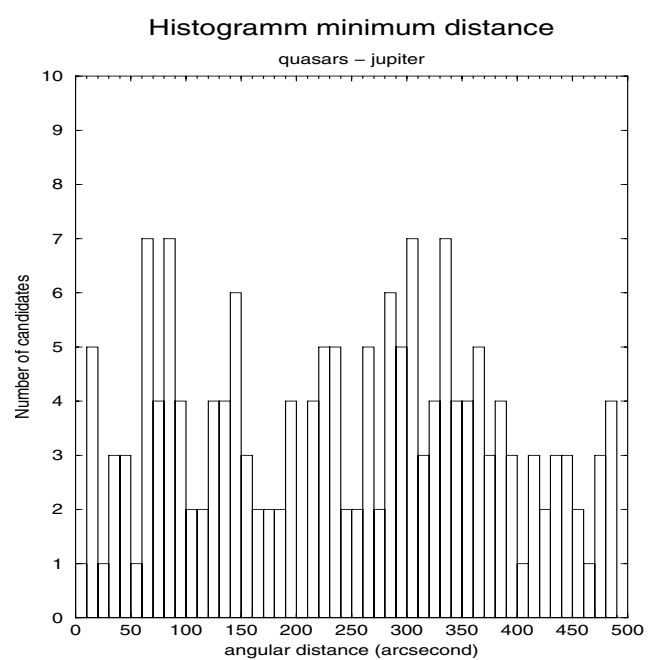

Fig. 7. The same histogram as in Fig. 6 but with a $10^{\prime \prime}$ bin.

close encounter as seen from the Earth and from Gaia, which can reach a few hours. The difference between Tables 1 and 2 is that the minimum value is given for the angular distance from the quasar to the jovicenter, in the first case, from the quasar to the planet limb in the second case. In order to compare these distances with the apparent angular size of the planet, we also give this last determination from the two sites (GAIA and the Earth), as the difference might reach a few 0.'01. In order to estimate the feasability of the observation from large ground-based telescopes as well as from GAIA, we also indicate the BRI photometry of the extragalactic sources concerned in Table 3.

\section{Orientation of Jupiter's axis of symmetry}

Knowing that Jupiter's flattening can have a significant and detectable influence on a light ray propagating in its vicinity (290 $\mu$ as for a grazing event, with the axis of symmetry perpendicular to the line of sight), and also to understand the relative position of Jupiter's equator (and consequently satellites) with respect to the trajectory of the extragalactic object, it is necessary to orient Jupiter's polar axis (which will be denoted by $\boldsymbol{k}$ in the next section) with respect to the observational point (a 
Table 1. List of the close encounters below 300" between Jupiter's geocenter and quasars during the time interval [2010-2015].

\begin{tabular}{|c|c|c|c|c|c|c|c|}
\hline $\begin{array}{l}\alpha \text { Quasar } \\
(\mathrm{J} 2000.0)\end{array}$ & $\begin{array}{r}\delta \text { quasar } \\
(\mathrm{J} 2000.0)\end{array}$ & $\begin{array}{r}\text { Date of event } \\
(\mathrm{JJ}) \\
\text { from the Earth }\end{array}$ & $\begin{array}{r}\text { Date of event } \\
(\mathrm{JJ}) \\
\text { from GAIA }\end{array}$ & $\begin{array}{r}\text { Min. ang. distance } \\
\text { to Jovicenter } \\
\text { from the Earth }\end{array}$ & $\begin{array}{r}\text { Min. distance } \\
\text { to Jovicenter } \\
\text { from GAIA } \\
\left({ }^{\prime \prime}\right)\end{array}$ & $\begin{array}{r}\text { Jupiter's angular } \\
\text { radius } \\
\text { from the Earth } \\
\left({ }^{\prime \prime}\right)\end{array}$ & $\begin{array}{r}\text { Jupiter's angular } \\
\text { radius } \\
\text { from GAIA } \\
\left({ }^{\prime \prime}\right)\end{array}$ \\
\hline 22.004544 & -13.151478 & 2010.01780 & 2010.01848 & 155.176916 & 155.254089 & 16.978 & 16.953 \\
\hline 23.889244 & -1.887272 & 2010.38923 & 2010.38775 & 167.507468 & 130.167585 & 18.899 & 18.881 \\
\hline 23.939442 & -1.605431 & 2010.40292 & 2010.40132 & 87.934831 & 50.321635 & 19.172 & 19.156 \\
\hline 0.071237 & -0.883578 & 2010.44433 & 2010.44205 & 136.620855 & 171.671447 & 20.067 & 20.063 \\
\hline 0.084132 & -0.746722 & 2010.44958 & 2010.44718 & 68.999806 & 34.444640 & 20.194 & 20.192 \\
\hline 0.172806 & -0.616947 & 2010.62936 & 2010.63130 & 284.587636 & 227.857326 & 24.326 & 24.388 \\
\hline 0.102228 & -1.116814 & 2010.66222 & 2010.66302 & 269.099724 & 219.105915 & 24.731 & 24.797 \\
\hline 0.076590 & -1.221850 & 2010.67157 & 2010.67226 & 30.732750 & 16.790082 & 24.813 & 24.879 \\
\hline 23.974428 & -1.885842 & 2010.70773 & 2010.70785 & 58.918990 & 95.006909 & 24.862 & 24.930 \\
\hline 0.213091 & 0.211056 & 2011.10347 & 2011.10415 & 264.465514 & 278.651688 & 17.262 & 17.234 \\
\hline 0.225157 & 0.304061 & 2011.10609 & 2011.10678 & 304.881822 & 319.359209 & 17.233 & 17.204 \\
\hline 0.275207 & 0.543294 & 2011.11636 & 2011.11693 & 7.033393 & 8.267031 & 17.123 & 17.094 \\
\hline 0.300059 & 0.767536 & 2011.12183 & 2011.12240 & 186.482566 & 202.050816 & 17.072 & 17.042 \\
\hline 0.333834 & 0.910314 & 2011.12845 & 2011.12902 & 82.056352 & 66.309867 & 16.999 & 16.970 \\
\hline 0.362024 & 1.144586 & 2011.13450 & 2011.13495 & 74.128831 & 89.920385 & 16.955 & 16.925 \\
\hline 0.357744 & 1.072380 & 2011.13336 & 2011.13393 & 70.330791 & 54.539730 & 16.955 & 16.925 \\
\hline 0.384206 & 1.259467 & 2011.13883 & 2011.13923 & 30.961777 & 15.201028 & 16.911 & 16.882 \\
\hline 0.398976 & 1.377727 & 2011.14191 & 2011.14237 & 37.241609 & 52.949135 & 16.891 & 16.862 \\
\hline 0.497807 & 2.101772 & 2011.16188 & 2011.16233 & 280.945030 & 295.586000 & 16.764 & 16.734 \\
\hline 0.617237 & 2.735175 & 2011.18423 & 2011.18458 & 198.850401 & 187.157316 & 16.654 & 16.624 \\
\hline 2.188849 & 12.176486 & 2012.12914 & 2012.13085 & 63.955222 & 77.236509 & 18.139 & 18.118 \\
\hline 2.189175 & 12.246211 & 2012.12959 & 2012.13130 & 292.234069 & 305.573110 & 18.139 & 18.117 \\
\hline 2.663587 & 14.559789 & 2012.23466 & 2012.23557 & 231.734197 & 229.621909 & 16.810 & 16.796 \\
\hline 3.416179 & 17.813728 & 2012.36893 & 2012.36915 & 144.496735 & 171.050821 & 16.796 & 16.769 \\
\hline
\end{tabular}

ground-based observer or GAIA). To achieve this, we represent this orientation by the intermediary of two parameters. $v$ represents the angle between the vertical direction (perpendicular to the celestial equator and Jupiter's polar axis, whereas $r_{\text {ratio }}$ represents the ratio of the angular distance between Jupiter's barycenter and Jupiter's visible pole, to Jupiter's apparent radius. These two parameters enable one to accurately give the position of Jupiter's visible pole in the plane perpendicular to the line of sight. Notice that Jupiter's polar axis is nearly perpendicular to its orbit (Jupiter's obliquity is $5^{\circ}$ ), whereas Jupiter's orbit inclination is rather small $\left(2^{\circ}\right)$, so that the angle between Jupiter's polar axis and the ecliptic should not exceed $7^{\circ}$. As our reference frame is equatorial, the angle $v$ should not exceed an amplitude greater than $30^{\circ}$ modulo $180^{\circ}$ according to which pole is visible (the south or the north one). The maximum is occurring when the angles above are summed together with the Earth's obliquity equal to $23^{\circ} 27^{\prime}$.

In Figs. 8 and 9 we represent the values of $v$ and $r_{\text {ratio, }}$, respectively, during the time interval 2005-2015. In Fig. 8 the sign of $v$ depends on the orientation of the polar axis with respect to the $\delta$ axis perpendicular to the equator. The sign is positive when it is oriented in the prograde direction and negative in the opposite case. The small oscillations with nearly annual frequency are the additional parallax effect due to the orbital motion of the Earth. The values of $r_{\text {ratio }}$ are always very close to 1 . In fact it never reaches an amplitude beyond the value $r_{\text {ratio }}=0.9982$. This means that Jupiter's axis of symmetry is permanently oriented almost perpendicularly to the line of sight (Fig. 9). The values $v=0^{\circ}$ and $v=180^{\circ}$ are reached respectively when three following axes are included in the same plane: Jupiter's polar axis, the axis corresponding to the line of sight and the $\delta$ axis. They correspond respectively to 2008 and 2013.5, the time interval between these two dates being logically half the revolution of Jupiter.

\section{Relativistic deflection of light}

At the mas and also sub-mas level, it is essential to take the relativistic deflection of light into account. For instance, Crosta $\&$ Mignard (2006) have recently proposed the determination of the PPN parameter $\gamma$ with GAIA in the frame of the GAREX project. The idea is dedicated to a small field experiment from observations close to the limb of a giant planet. Concerning the theoretical framework, let us emphasize that light deflection in the gravitational field of an axisymmetric body is nowadays well understood. Klioner (1989), Klioner \& Kopeikin (1992), and Kopeikin (1997) presented an integration of null geodesic equations leading to a complete explicit solution for the influence of the mass quadrupole moment. In another approach (Le Poncin-Lafitte et al. 2004; Le Poncin-Lafitte \& Teyssandier 2005) explicit calculations of the influence of all mass multipole moments are obtained from the determination of the time transfer function between two points located at a finite distance. Let us introduce the key points of this last formalism. Consider an axisymmetric massive body of mass $M$, namely Jupiter. The center of mass $\mathrm{O}$ of this body is chosen as the origin of a quasiCartesian coordinates $\left(c t, \boldsymbol{x}=\left\{x^{i}, i=1,2,3\right\}\right)$ and we choose the axis of symmetry as the $x^{3}$-axis ( $\boldsymbol{k}$ is the unit vector along this axis). Let $\Gamma$ be a light ray emitted at event $x_{\mathrm{A}}=\left(c t_{\mathrm{A}}, \boldsymbol{x}_{\mathrm{A}}\right)$ and received by a given observer located at $x_{\mathrm{B}}=\left(c t_{\mathrm{B}}, \boldsymbol{x}_{\mathrm{B}}\right)$. The vector tangent to $\Gamma$ at $x_{\mathrm{B}}$ is given by

$l_{x_{\mathrm{B}}}=N_{\mathrm{AB}}+\lambda\left(x_{\mathrm{A}}, \boldsymbol{x}_{\mathrm{B}}\right)$

where

$N_{\mathrm{AB}}=\frac{x_{\mathrm{B}}-x_{\mathrm{A}}}{\left|x_{\mathrm{B}}-x_{\mathrm{A}}\right|}$, 
Table 2. List of the close encounters below 300" between Jupiter's limb and quasars during the time interval [2010-2015].

\begin{tabular}{|c|c|c|c|c|c|c|c|}
\hline $\begin{array}{l}\alpha \text { Quasar } \\
(\mathrm{J} 2000.0)\end{array}$ & $\begin{array}{r}\delta \text { quasar } \\
(\mathrm{J} 2000.0)\end{array}$ & $\begin{array}{r}\text { Date of event } \\
(\mathrm{JJ}) \\
\text { from the Earth }\end{array}$ & $\begin{array}{r}\text { Date of event } \\
(\mathrm{JJ}) \\
\text { from GAIA }\end{array}$ & $\begin{array}{r}\text { Min. ang. distance } \\
\text { to Jupiter limb } \\
\text { from the Earth } \\
\left({ }^{\prime \prime}\right)\end{array}$ & $\begin{array}{r}\text { Min. ang. distance } \\
\text { to Jupiter limb } \\
\text { from GAIA } \\
\left({ }^{\prime \prime}\right)\end{array}$ & $\begin{array}{r}\text { Jupiter's angular } \\
\text { radius } \\
\text { from the Earth } \\
\left({ }^{\prime \prime}\right)\end{array}$ & $\begin{array}{r}\text { Jupiter's angular } \\
\text { radius } \\
\text { from GAIA } \\
\left({ }^{\prime \prime}\right)\end{array}$ \\
\hline 22.004544 & -13.151478 & 2010.01780 & 2010.01848 & 138.198482 & 138.300322 & 16.978 & 16.953 \\
\hline 23.889244 & -1.887272 & 2010.38923 & 2010.38775 & 148.608110 & 111.286424 & 18.899 & 18.881 \\
\hline 23.939442 & -1.605431 & 2010.40292 & 2010.40132 & 68.762967 & 31.165017 & 19.172 & 19.156 \\
\hline 0.071237 & -0.883578 & 2010.44433 & 2010.44205 & 116.553802 & 151.608470 & 20.067 & 20.063 \\
\hline 0.084132 & -0.746722 & 2010.44958 & 2010.44718 & 48.805528 & 14.252588 & 20.194 & 20.192 \\
\hline 0.172806 & -0.616947 & 2010.62936 & 2010.63130 & 260.261639 & 203.469030 & 24.326 & 24.388 \\
\hline 0.102228 & -1.116814 & 2010.66222 & 2010.66302 & 244.368853 & 194.308181 & 24.731 & 24.797 \\
\hline 0.076590 & -1.221850 & 2010.67157 & 2010.67226 & 5.920671 & -8.089766 & 24.813 & 24.879 \\
\hline 23.974428 & -1.885842 & 2010.70773 & 2010.70785 & 34.057019 & 70.076585 & 24.862 & 24.930 \\
\hline 0.213091 & 0.211056 & 2011.10347 & 2011.10415 & 247.202958 & 261.418026 & 17.262 & 17.234 \\
\hline 0.225157 & 0.304061 & 2011.10609 & 2011.10678 & 287.648288 & 302.154669 & 17.233 & 17.204 \\
\hline 0.275207 & 0.543294 & 2011.11636 & 2011.11693 & -10.090172 & -8.827255 & 17.123 & 17.094 \\
\hline 0.300059 & 0.767536 & 2011.12183 & 2011.12240 & 169.410335 & 185.007941 & 17.072 & 17.042 \\
\hline 0.333834 & 0.910314 & 2011.12845 & 2011.12902 & 65.056574 & 49.339506 & 16.999 & 16.970 \\
\hline 0.362024 & 1.144586 & 2011.13450 & 2011.13495 & 57.174328 & 72.995317 & 16.955 & 16.925 \\
\hline 0.357744 & 1.072380 & 2011.13336 & 2011.13393 & 53.376288 & 37.614663 & 16.955 & 16.925 \\
\hline 0.384206 & 1.259467 & 2011.13883 & 2011.13923 & 14.050135 & -1.681168 & 16.911 & 16.882 \\
\hline 0.398976 & 1.377727 & 2011.14191 & 2011.14237 & 20.350493 & 36.087471 & 16.891 & 16.862 \\
\hline 0.497807 & 2.101772 & 2011.16188 & 2011.16233 & 264.180837 & 278.851344 & 16.764 & 16.734 \\
\hline 0.617237 & 2.735175 & 2011.18423 & 2011.18458 & 182.195893 & 170.532510 & 16.654 & 16.624 \\
\hline 2.188849 & 12.176486 & 2012.12914 & 2012.13085 & 45.815842 & 59.118567 & 18.139 & 18.118 \\
\hline 2.189175 & 12.246211 & 2012.12959 & 2012.13130 & 274.248325 & 287.455599 & 18.139 & 18.117 \\
\hline 2.663587 & 14.559789 & 2012.23466 & 2012.23557 & 214.923734 & 212.835625 & 16.810 & 16.369 \\
\hline 3.416179 & 17.813728 & 2012.36893 & 2012.36915 & 128.100832 & 154.680989 & 16.396 & 16.369 \\
\hline
\end{tabular}

and

$\lambda\left(x_{\mathrm{A}}, x_{\mathrm{B}}\right)=\lambda_{M}\left(x_{\mathrm{A}}, x_{\mathrm{B}}\right)+\sum_{n=2}^{\infty} \lambda_{J_{n}}\left(x_{\mathrm{A}}, x_{\mathrm{B}}\right)$.

The quantity $\lambda_{M}\left(x_{\mathrm{A}}, \boldsymbol{x}_{\mathrm{B}}\right)$ is well known as the deflection of light due to mass $M$. Its expression can be found in any reference textbook concerning general relativity (Weinberg 1972; Misner Thorne \& Wheeler 1973; Will 1993). We can determine explicitely $\lambda_{J_{n}}\left(\boldsymbol{x}_{\mathrm{A}}, \boldsymbol{x}_{\mathrm{B}}\right)$ by the use of orthogonal polynomials, i.e. Legendre and Gegenbauer functions (see Le Poncin-Lafitte \& Teyssandier 2005). But here, as in Crosta \& Mignard (2006), we only consider the influence of the mass and mass-quadrupole moment of Jupiter on light propagation with the emission event of the light ray located at spatial infinity. In this case, the radial variable $r_{\mathrm{A}}=\left|\boldsymbol{x}_{\mathrm{A}}\right|$ increases indefinitely, and the unit direction of the quasar becomes the fixed vector $\boldsymbol{N}$ at spatial infinity. Moreover, it is clear that

$\lim _{r_{A} \rightarrow \infty} N_{\mathrm{AB}}=\boldsymbol{N}$.

The vector $\lambda\left(x_{\mathrm{A}}, \boldsymbol{x}_{\mathrm{B}}\right)$ approaches a limit that can be considered as a function of $\boldsymbol{N}$ and $\boldsymbol{x}_{\mathrm{B}}$. As a consequence, we henceforth put:

$\lambda\left(N, x_{\mathrm{B}}\right)=\lim _{r_{A} \rightarrow \infty} \lambda\left(x_{\mathrm{A}}, x_{\mathrm{B}}\right)$

By taking Eqs. (5) and (4) into account, $\lambda\left(\boldsymbol{N}, \boldsymbol{x}_{\mathrm{B}}\right)$ may be expanded as

$\lambda\left(N, x_{\mathrm{B}}\right)=\lambda_{M}\left(N, x_{\mathrm{B}}\right)+\sum_{n=2}^{\infty} \lambda_{J_{n}}\left(N, x_{\mathrm{B}}\right)$,

where

$$
\begin{aligned}
& \lambda_{M}\left(N, x_{\mathrm{B}}\right)=\lim _{r_{A} \rightarrow \infty} \lambda_{M}\left(x_{\mathrm{A}}, x_{\mathrm{B}}\right), \\
& \lambda_{J_{n}}\left(N, x_{\mathrm{B}}\right)=\lim _{r_{A} \rightarrow \infty} \lambda_{J_{n}}\left(\boldsymbol{x}_{A}, \boldsymbol{x}_{\mathrm{B}}\right) .
\end{aligned}
$$

By defining $\alpha, r_{\mathrm{c}}$, and the unit vector $\boldsymbol{q}_{N}$ as

$\cos \alpha=N, . \frac{x_{\mathrm{B}}}{\left|\boldsymbol{x}_{\mathrm{B}}\right|}$,

$r_{\mathrm{c}}=2\left|x_{\mathrm{B}}\right| \cos \left(\frac{\alpha}{2}\right) \sin \left(\frac{\alpha}{2}\right)$,

and

$\boldsymbol{q}_{N}=\frac{\boldsymbol{n}_{\mathrm{B}}-\boldsymbol{N}}{\sqrt{2\left(1-\boldsymbol{N} \cdot \boldsymbol{n}_{\mathrm{B}}\right)}}=\left(2 \sin \frac{\alpha}{2}\right)^{-1}\left(\boldsymbol{n}_{\mathrm{B}}-\boldsymbol{N}\right)$,

we can deduce from the explicit expression of $\lambda\left(x_{\mathrm{A}}, \boldsymbol{x}_{\mathrm{B}}\right)$ obtained by Le Poncin-Lafitte \& Teyssandier (2005) approximate formulae for $\lambda_{M}\left(N, x_{\mathrm{B}}\right)$ and $\lambda_{\mathrm{J}_{2}}\left(\boldsymbol{N}, \boldsymbol{x}_{\mathrm{B}}\right)$ as follows

$$
\begin{aligned}
& \lambda_{M}\left(\boldsymbol{N}, \boldsymbol{x}_{\mathrm{B}}\right) \approx(\gamma+1) \frac{G M}{c^{2} r_{\mathrm{B}}} \frac{\frac{\boldsymbol{x}_{\mathrm{B}}}{\left|\boldsymbol{x}_{\mathrm{B}}\right|}-\boldsymbol{N}}{1-\boldsymbol{N} \cdot \frac{\boldsymbol{x}_{\mathrm{B}}}{\left|\boldsymbol{x}_{\mathrm{B}}\right|}} \\
& \lambda_{\mathrm{J}_{2}}\left(\boldsymbol{N}, \boldsymbol{x}_{\mathrm{B}}\right) \approx(\gamma+1) \frac{2 G M}{c^{2} r_{\mathrm{c}}} J_{2}\left(\frac{r_{\mathrm{e}}}{r_{\mathrm{c}}}\right)^{2} \cos ^{3}\left(\frac{\alpha}{2}\right) \\
& \quad \times\left\{\left[1-\left(\boldsymbol{k} \cdot \frac{\boldsymbol{x}_{\mathrm{B}}}{\left|\boldsymbol{x}_{\mathrm{B}}\right|}\right)^{2}-4\left(\boldsymbol{k} \cdot \boldsymbol{q}_{N}\right)^{2}\right] \boldsymbol{q}_{N}\right. \\
&\left.-2\left(\boldsymbol{k} \cdot \boldsymbol{q}_{N}\right)\left[\left(\boldsymbol{k} \cdot \frac{\boldsymbol{x}_{\mathrm{B}}}{\left|\boldsymbol{x}_{\mathrm{B}}\right|}\right) \frac{\boldsymbol{x}_{\mathrm{B}}}{\left|\boldsymbol{x}_{\mathrm{B}}\right|}-\boldsymbol{k}\right]\right\},
\end{aligned}
$$

$r_{\mathrm{e}}$ being the equatorial radius of the planet. We show in the following the amplitude of the deflection of light for chosen close approach events.

\section{Specific description of several close encounters}

We operate a selection of the most interesting close-encounter events between Jupiter and our set of quasars with the particular aim of showing a large deflection of light due to the 
Table 3. BRI Magnitudes of the quasars subject to close encounters with Jupiter in the Tables 1 and 2.

\begin{tabular}{|c|c|c|c|c|c|c|}
\hline $\begin{array}{l}\alpha \text { Quasar } \\
(\mathrm{J} 2000.0)\end{array}$ & $\begin{array}{r}\delta \text { quasar } \\
(\mathrm{J} 2000.0)\end{array}$ & $\begin{array}{r}\text { Date of event } \\
(\mathrm{JJ}) \\
\text { from the Earth }\end{array}$ & $\begin{array}{r}\text { Date of event } \\
(\mathrm{JJ}) \\
\text { from GAIA }\end{array}$ & B & $\mathrm{R}$ & I \\
\hline 22.004544 & -13.151478 & 2010.01780 & 2010.01848 & 18.29 & 18.26 & 17.15 \\
\hline 23.889244 & -1.887272 & 2010.38923 & 2010.38775 & 18.28 & 17.87 & 18.23 \\
\hline 23.939442 & -1.605431 & 2010.40292 & 2010.40132 & 16.27 & 16.04 & 15.67 \\
\hline 0.071237 & -0.883578 & 2010.44433 & 2010.44205 & 19.94 & 19.72 & - \\
\hline 0.084132 & -0.746722 & 2010.44958 & 2010.44718 & 20.29 & 20.03 & - \\
\hline 0.172806 & -0.616947 & 2010.62936 & 2010.63130 & 19.22 & 18.27 & 17.93 \\
\hline 0.102228 & -1.116814 & 2010.66222 & 2010.66302 & 20.30 & 19.86 & 17.05 \\
\hline 0.076590 & -1.221850 & 2010.67157 & 2010.67226 & 18.65 & 18.67 & 17.56 \\
\hline 23.974428 & -1.885842 & 2010.70773 & 2010.70785 & 17.44 & 16.75 & 16.22 \\
\hline 0.213091 & 0.211056 & 2011.10347 & 2011.10415 & 18.80 & 18.97 & 17.89 \\
\hline 0.225157 & 0.304061 & 2011.10609 & 2011.10678 & 19.04 & 19.25 & 18.26 \\
\hline 0.275207 & 0.543294 & 2011.11636 & 2011.11693 & 19.39 & 19.47 & 18.31 \\
\hline 0.300059 & 0.767536 & 2011.12183 & 2011.12240 & 18.49 & 18.84 & 18.20 \\
\hline 0.333834 & 0.910314 & 2011.12845 & 2011.12902 & 19.40 & 18.58 & 18.35 \\
\hline 0.362024 & 1.144586 & 2011.13450 & 2011.13495 & 18.91 & 19.52 & 18.41 \\
\hline 0.357744 & 1.072380 & 2011.13336 & 2011.13393 & 18.10 & 18.54 & 18.04 \\
\hline 0.384206 & 1.259467 & 2011.13883 & 2011.13923 & 18.61 & 18.43 & 17.45 \\
\hline 0.398976 & 1.377727 & 2011.14191 & 2011.14237 & 18.23 & 17.96 & 18.01 \\
\hline 0.497807 & 2.101772 & 2011.16188 & 2011.16233 & 17.71 & 17.78 & 17.41 \\
\hline 0.617237 & 2.735175 & 2011.18423 & 2011.18458 & 18.84 & 18.49 & 18.46 \\
\hline 2.188849 & 12.176486 & 2012.12914 & 2012.13085 & 19.03 & 18.94 & 18.11 \\
\hline 2.189175 & 12.246211 & 2012.12959 & 2012.13130 & 19.99 & 19.00 & 18.71 \\
\hline 2.663587 & 14.559789 & 2012.23466 & 2012.23557 & 18.65 & 18.74 & - \\
\hline 3.416179 & 17.813728 & 2012.36893 & 2012.36915 & 17.43 & 16.29 & 14.98 \\
\hline
\end{tabular}

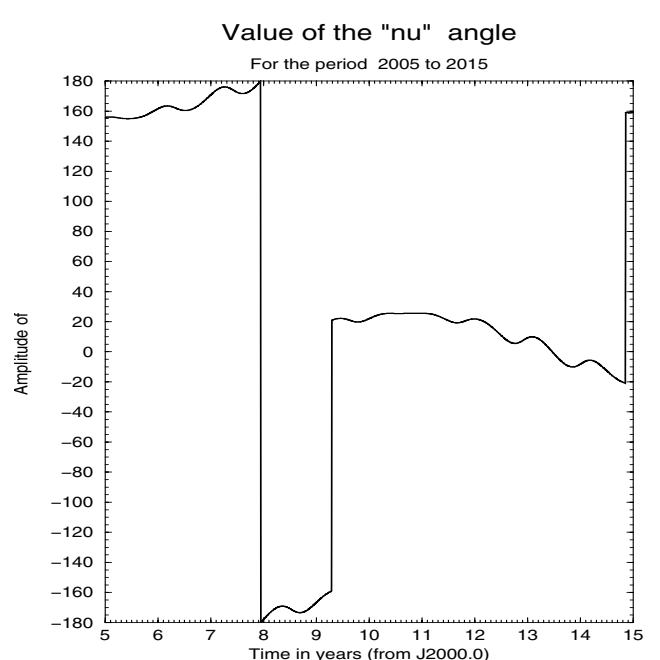

Fig. 8. Variations in the angle $v$.

relativistic effect as modeled in the preceding section. Our selection was taken from the 23 encounters for which the angular distance between Jupiter's limb and the quasars goes below $5^{\prime}$, (i.e. $300^{\prime \prime}$ ), occurring in a rather small time interval between 2010.0 and 2012.5. These events are listed in Tables 1 and 2, which respectively give the minimum angular distance to the Jupiter's center and limb, with the corresponding close approach dates, when observed both from the Earth and from the GAIA satellite. Jupiter's radius as seen from the two observing sites is also given as a supplementary indication. In the following, two events with the associated relativistic effects are shown in Figs. 10-13.

For the first event, occurring at 2010.671 and represented in Fig. 10, the quasar as seen from GAIA is crossing the Jupiter disk (occultation), whereas it is a grazing event from an

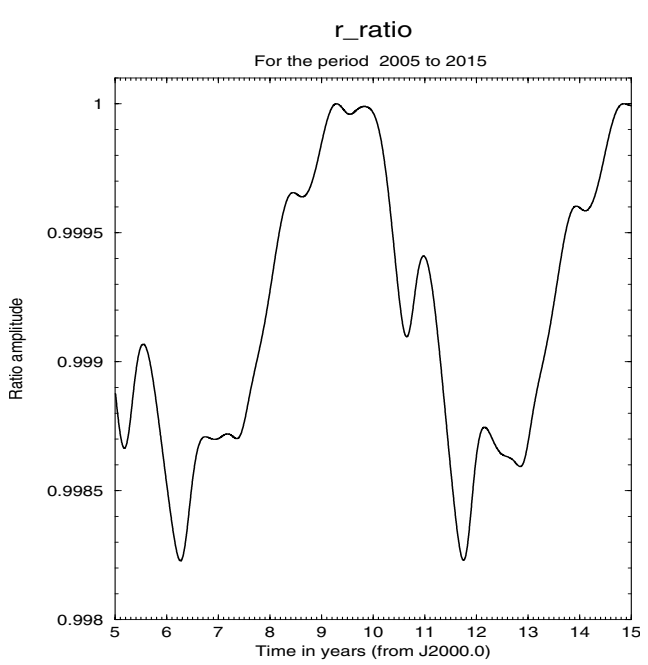

Fig. 9. Variations in the ratio $r_{\text {ratio }}$.

Earth-based observer, with a minimum angular distance to the limb of $6^{\prime \prime}$. The corresponding deflection of light is represented in Fig. 11. Of course it reaches the maximum value of $0.0162^{\prime \prime}$ (i.e. 16.2 mas) when the quasar is located just at the planet's limb. In fact because of the curvature of the beam, the quasar should be still visible, whereas it is located geometrically behind the planet. Notice that at the closest distance of 6 " when observed from the Earth, the relativistic effect still reaches 0 . $^{\prime} 014$ and also that the time interval of $6.9 \times 10^{-4} \mathrm{y}$ between the close approaches from the two sites corresponds to $0.25 \mathrm{~d}$. The small segment at the top of the GAIA curve in Fig. 11 corresponds to the short interval of time of the occultation for which no signal exists.

For the second event (Figs. 12 and 13) chosen at 2011.117, the quasar is undergoing an occultation both from the Earth and 


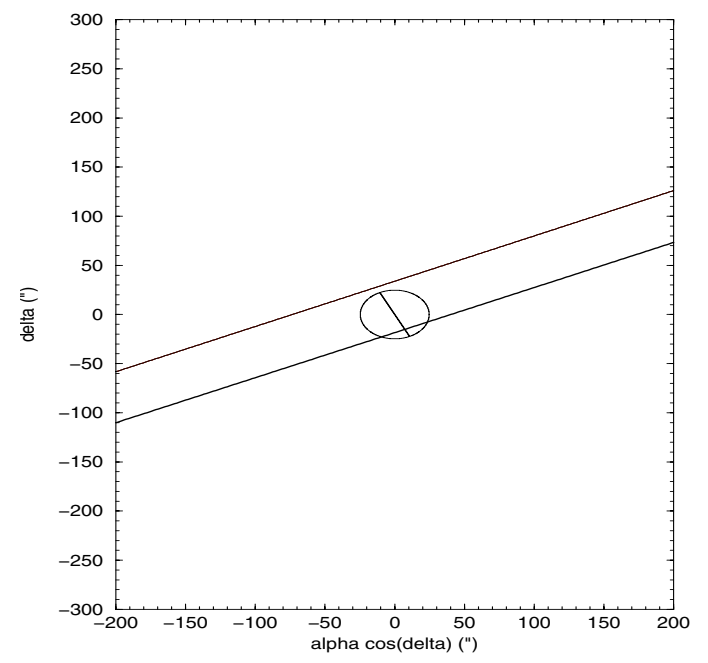

Fig. 10. Close approach between the quasar with coordinates $\alpha=$ $0^{\mathrm{h}} 04^{\mathrm{mn}} 35.7^{\mathrm{sec}}$ and $\delta=-1^{\circ} 13^{\prime} 19^{\prime \prime}$ and Jupiter.

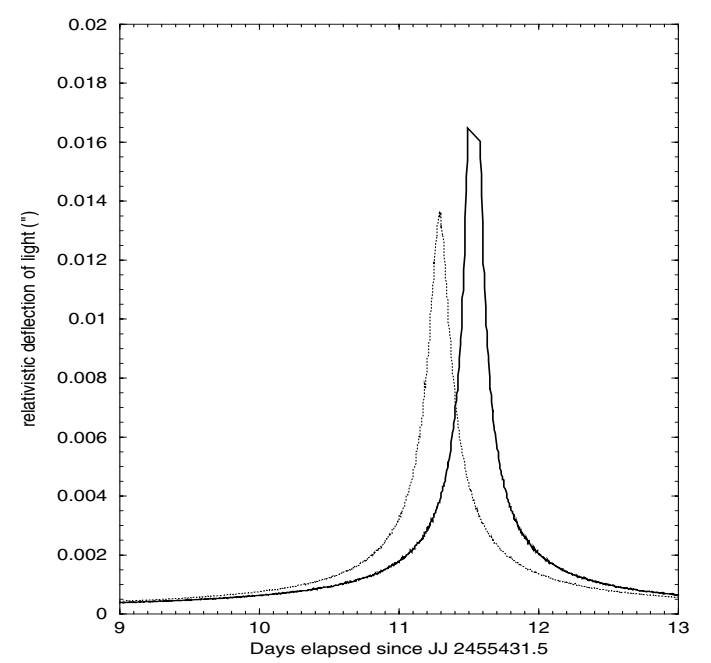

Fig. 11. Relativistic deflection of light of the quasar with coordinates and $\alpha=0^{\mathrm{h}} 04^{\mathrm{mn}} 35.7^{\mathrm{sec}}$ and $\delta=-1^{\circ} 13^{\prime} 19^{\prime \prime}$, during the close approach with Jupiter.

from the astrometric satellite. Notice that the angular diameter of Jupiter is considerably smaller than in the preceding case (the angular radius is 17 '. $^{\prime} 1$ instead of $24 ., 8$ as can be checked in Table 1), because Jupiter's synodic configuration is far from the opposition. We can also verify that in these two close approaches, Jupiter's axis, represented by a segment, is quasi perpendicular to the relative trajectory of the quasars. Indeed, this constitutes a clear advantage from an astrometric point of view, for it means that the quasar crossing line is parallel to Jupiter's equator; therefore, differential astrometric measurements with the majority of the satellites having a near zero inclination will be very efficient.

We can observe that in the two cases, the amplitude of the deflection of the beam is quite large, with a maximum value of 16 milliarcseconds. This is an encouraging point for proving the capacity of detecting the relativistic effects of Jupiter on remote light rays.

\section{Conclusion and future prospects}

In view of the increasing number of recorded quasars in optical catalogues (Véron-Cetty \& Véron 2003) we found that it was

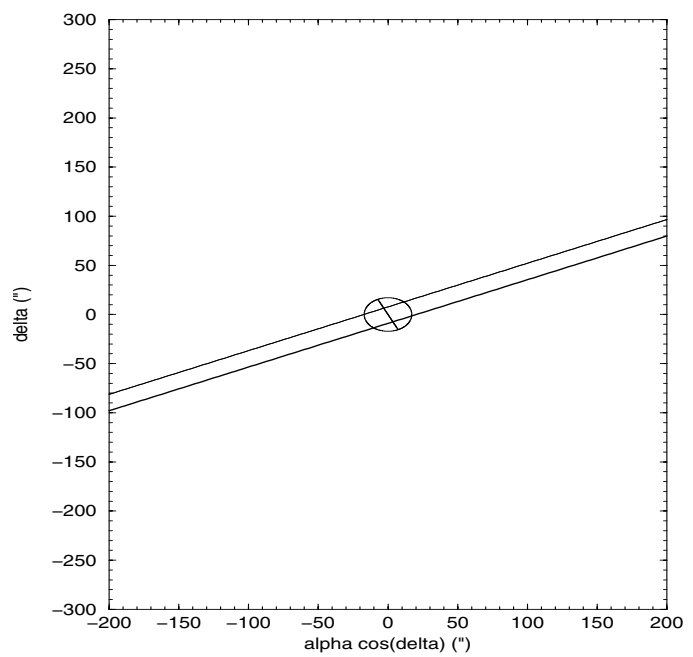

Fig. 12. Close approach between the quasar with coordinates $\alpha=$ $0^{\mathrm{h}} 16^{\mathrm{mn}} 30.7^{\mathrm{sec}}$ and $\delta=0^{\circ} 32^{\prime} 36^{\prime \prime}$ and Jupiter.

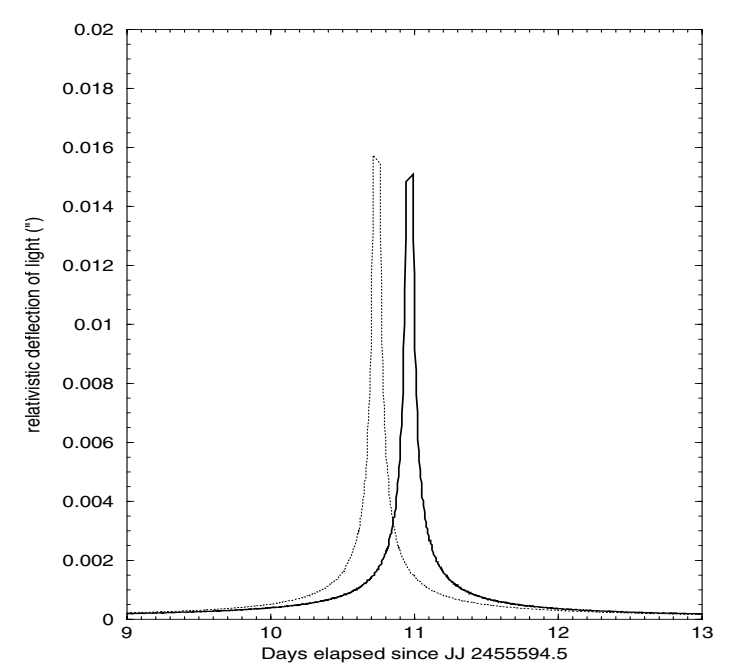

Fig. 13. Relativistic deflection of light of the quasar with coordinates $\alpha=0^{\mathrm{h}} 16^{\mathrm{mn}} 30.7^{\mathrm{sec}}$ and $\delta=0^{\circ} 32^{\prime} 36^{\prime \prime}$, during the close approach with Jupiter.

interesting to determine the close approaches between a planet like Jupiter and the recorded quasars as precisely as possible and for a rather large time interval (10 years extending between 2005 and 2015) including the very promising astrometric GAIA space mission. Our study was mainly motivated by how pointlike extragalactic objects as quasars might be playing a leading role in the coming years or decades as absolute astrometric standards, replacing calssical astrometric standards such as Hipparcos stars, which are subject to uncertainties in their proper motions that lead to gradually deteriorating astrometric determinations. Moreover with the goal of accurately determining the link between the Dynamical Reference System materialized by the motions of the planets and a quasi-inertial Reference System as the ICRS, which is one of the leading challenges of modern astrometry, the study carried out in the present paper might be quite useful. Our main results are:

- Statistically we found a substantial number of close encounters between Jupiter and the quasars recorded by (Véron-Cetty $\&$ Véron 2003) during the interval of time 2010-2015, i.e. 232 phenomena at all, up to 10' both in $\alpha \cos \delta$ and in $\delta$. In particular, we could select 23 phenomena during the period 
2010-2012 corresponding more or less to the GAIA space mission, with an upper limit of $5^{\prime}$.

- Considering that these phenomena are interesting not only from the GAIA satellite, but also from ground-based telescopes, we have modeled the parallax effect in bi-dimensional coordinates $(\alpha \cos \delta, \delta)$ between the two sites. Undoubtedly, differential observations of the same phenomena between the two sites (the Earth and GAIA) might be very interesting in an astrometric point of view, as given by the parallax effect above. Moreover, we have given a very precise way to position the figure axis of Jupiter with respect to the observer, showing that this axis is quasi-permanently directed perpendicularly to the line of sight of the planet.

- Concerning the relativistic light deflection, we only focused on approximate formulae that more or less describe the influence of the mass-monopole and the mass-quadrupole of Jupiter. In order to be able to calculate the effect due to $J_{2}$, we have determined the figure axis orientation of Jupiter. In the future, the complete formalism of Le Poncin-Lafitte and Teyssandier (2005) can be used, if necessary, to take the influence of higher mass-multipole moments on light deflection into account.

- In the case of two interesting single examples of grazing phenomena, we have shown the order of magnitude of the light deflection related to them, with a maximum amount of roughly 16 mas. At the same time we have shown the differential positioning between the quasar and Jupiter when observed both from the Earth and from GAIA, and the difference in deflection between the two sites.

This study may be extended to other planets (as Mars and Saturn) and to an much larger number of recorded quasars, as the number of these objects is increasing considerably, thanks to the arrival and extension of new survey catalogues. Moreover in the case of Jupiter and Saturn, the large number of satellites should naturally lead to a large number of close approaches between these satellites and the quasars recorded in this paper. Therefore a suitable astrometric reduction of close encounter events between these extragalactic objects and the planets will be very precious in insuring the link between the dynamical system and the ICRS (International Celestial Reference System).

\section{References}

Adelman-McCarthy, J. K., Agueros, M. A., Allam, S. S., et al. 2006, ApJS, 162, 38

Bienaymé, O., \& Turon, C., eds. 2002, GAIA: A European Space Project (Les Ulis: EDP Sciences)

Boboltz, D. A., Fey, A. L., Johnston, K. J., et al. 2003, AJ, 126, 484

Croom, S. M., Smith, R. J., Boyle, B. J., et al. 2001, MNRAS, 322, L29

Crosta, M. T., \& Mignard, F. 2006, Class. Quant. Gravity, 23, 15, 4853

Da Silva Neto, D. N, Assafin, M., Andrei, A. H., \& Vieira Martins, R. 2005, Proccedings of the Gaia Symposium The three-dimensional Universe with Gaia, ed. C. Turon, K. S. O'Flaherty, \& M. A. C. Perryman, ESA SP-576, 285

Diolaiti, E., Bendinelli, O., Bonaccini, D., et al. 2000, ASSS, 147, 335

ESA 2000, GAIA: Composition, Formation and Evolution of the Galaxy, Concept and Technology Study Report, ESA-SCI[2000]4 (Noordwijk: ESA)

Fey, A. L., Boboltz, D. A., Gaume, R. A., \& Johnston, K. J. 2006, AJ, 131, 1084

Klioner, S. A. 1989, Soobschch. Inst. Prik. Astron. No. 6 (Communications of the Institute of Applied Astronomy, No. 6, St. Petersburg, in Russian)

Klioner, S. A. 2003, AJ, 125, 1580

Klioner, S. A., \& Kopeikin, S. M. 1992, AJ, 104, 897

Kopeikin, S. M. 1997, J. Math. Phys., 38, 2587

Le Poncin-Lafitte, C., Linet, B., \& Teyssandier, P. 2004, Class. Quant. Gravity, 21, 18, 4463

Le Poncin-Lafitte, C., \& Teyssandier, P. 2005, Journée Systèmes de Référence Spatio-temporels 2004, Observatoire de Paris, Ed. N. Capitaine, ISBN2901057-51-9, 204

Misner, C. W., Thorne, K. S., \& Wheeler, J. A. 1973, Gravitation (W. H. Freeman)

Perryman, M. A. C., et al. 2001, A\&A, 369, 339

Schneider, D. P., Richards, G. T., Fan, X., et al. 2002, AJ, 123, 567

Souchay, J., Gontier, A. M., \& Barache, C. 2006, A\&A, 453, 743

Véron-Cetty, M. P., \& Véron, P. 2001, A\&A, 374, 92

Véron-Cetty, M. P., \& Véron, P. 2003, A\&A, 412, 399

Weinberg, S. 1972, Gravitation and Cosmology: Principles and Applications of the General Theory of Relativity (John Wiley \& Sons)

Will, C. M. 1993, Theory and experiment in gravitational physics, (rev. ed.; Cambridge: Cambridge University Press) 